\title{
LA EDUCACIÓN AMBIENTAL: ¿UNO DE LOS MEDIOS PARA EVITAR LOS DESASTRES DE ORIGEN ANTRÓPICO?
}

\author{
Dra. Alexa Jengich Buck ${ }^{1}$
}

\section{Resumen}

Este artículo busca reflexionar sobre el impacto que tiene la presencia del ser humano en su medio ambiente, lo que ocasiona con frecuencia desastres catalogados como de origen antrópico.

Además, se parte de la urgencia de revisar algunos aspectos que interfieren con la educación ambiental y que ponen en riesgo la sobrevivencia de una generación joven que ya nació.

En consecuencia, interesa enfocar el problema desde el punto de vista epistemológico, analizando el asunto desde la óptica de aquellos pensamientos que mantienen y hacen difícil un cambio de la educación ambiental, hacia el manejo preventivo de las amenazas para el bienestar de toda la población. Se utiliza brevemente el ejemplo del manejo del recurso hídrico, para plantear parte de la dinámica de los desastres de origen antrópico.

\section{Abstract}

The author deals with the human impact over its surrounding, which frecuently give rise to ecological disasters known as of anthropic origen.

It is considered urgent to review some aspects which interfere with environmental education, putting at risk the survival of young generation already born. In consequence, it is of interest to focus the problem from the epistemological point of view, placing special attention to those thoughts which makes it hard to work the environmental education towards preventing situations which threatens the population wellbeing. To that goal the water management example is briefly used to present part of disasters of anthropic origen.

Palabras claves: Desastres de origen antrópico, educación ambiental, desarrollo

1 Profesora investigadora del Centro de Investigación y Docencia en Educación (CIDE), Universidad Nacional. Licenciada en Psicología; máster en Administración de Negocios con énfasis en mercadeo y doctora en Estudios Latinoamericanos con énfasis en pensamiento latinoamericano. 
humano, explotación de recursos naturales, agua, inundaciones, crecimiento demográfico, pensamiento económico y religioso.

$\mathrm{S}$ e podría empezar este artículo tratando de definir los desastres de origen antrópico, como aquellos desastres naturales que se originan por acciones del ser humano, que causan pérdidas materiales cuantificables y daños irreparables para las personas. Estos desastres pueden ser considerados como producto de la explotación de los recursos naturales más allá de su capacidad de regeneración, en caso de que esta sea posible.

En consecuencia, surge la interrogante de por qué se dan estas situaciones catastróficas, cuando se tienen al alcance los medios para evitarlas. A raíz de lo anterior, interesa profundizar en algunos tipos de pensamiento que provocan este comportamiento del ser humano, con miras a construir un marco epistemológico que permita una educación ambiental sustentada en una conceptualización diferente, tanto del desarrollo humano como de la Naturaleza. A su vez, esto implica explorar pensamientos alternativos que provienen de múltiples áreas del conocimiento.

A manera de ejemplo, se analiza aquí muy brevemente, el manejo que se hace del recurso hídrico en algunas zonas de este país, que pueden llevar a una carestía total de agua o a una abundancia sin control, como ocurre en el caso de ciertos tipos de inundaciones que se pueden catalogar como desastres de origen antrópico.

Para empezar, se puede plantear el hecho de que vivimos en un sistema socioeconómico en el cual gran parte de la conducta humana está orientada al lucro y a la acumulación de dinero, lo que la sociedad ha llegado a manejar como símbolo de éxito o desarrollo personal. Dentro de esta perspectiva, el fin justifica cualquier medio que se utilice para obtener las ganancias, aunque se provoquen daños ambientales.

Este modelo a su vez se basa en la ley del más fuerte, por lo que las necesidades colectivas quedan supeditadas a los intereses individualistas. En consecuencia, dentro de esta perspectiva, cualquier preocupación de índole ecológica que desee el bienestar de la toda la población, se convierte en un desafío a esa dinámica del manejo del poder. Esto se evidencia en comportamientos que buscan descalificar a aquellos a quienes les preocupa el deterioro del medio ambiente con frases tales como: “¡Ah, esos ecologistas!”; expresión despectiva que se escucha con frecuencia desde esta perspectiva economicista. Tales reacciones se podrían explicar como un intento de excluir del 
discurso dominante todas aquellas ideas o acciones que se puedan interpretar como algo que atenta al orden económico establecido.

Sin embargo, siguiendo este mismo ejemplo, fácilmente se aprecian serias contradicciones en el sistema, pues actualmente también se utiliza la palabra "ecológico" para vender un pedazo de tierra o para anunciar un hotel de montaña. Sin profundizar en lo que implican tales mensajes, es importante enfatizar que, implícitamente, se está reconociendo que un enfoque ecológico es algo indispensable para lograr el bienestar, en este caso en términos de descanso o garantizar la salud.

Dentro del enfoque anterior, es importante agregar que existe la expresión: "vos que sos ecologista, arreglá ese problema...", cuando se trata de defender un recurso natural. Pareciera, entonces, que el asunto de defender la sostenibilidad del medio, sólo fuera del interés de unos pocos. Además, esta actitud se asocia con algunos vínculos utilitaristas que se establecen entre las personas, en los que se confunde el bienestar con una tenencia de cosas, en un mundo consumista en que todo se desecha cuando "ya no sirve", sean estos animales, plantas o miembros de la misma especie; algunos han denominado a este hecho como una cultura de muerte y no de vida.

En consecuencia, se puede mencionar aquí gran cantidad de amenazas a los recursos naturales que no se abordan lo suficiente o su enfoque es parcial. Por ejemplo, cuesta encontrar en los textos que se utilizan para la educación ambiental algunas referencias en las cuales se analice el problema en forma interrelacionada, como que el análisis de los deslizamientos o la infertilidad - de la tierra se realice tomando en consideración los aspectos psicosociales que aquí se mencionan. Se puede agregar que al presentarse la deforestación, producto de un consumo desmedido de la madera o de la necesidad de cultivar grandes extensiones para satisfacer una demanda de alimentos, no se hace mención del aumento geométrico de la población como la causa de fondo.

Se podría afirmar que la integración conceptual es excluida de la agenda educativa, pues esto demanda una educación ambiental de carácter interdisciplinario que se oriente a evitar una conducta humana de explotación insostenible de los recursos.

No se puede dejar de citar aquí a Thomas R. Malthus, quien desde finales del siglo XVIII ya visualizaba los desfaces entre el crecimiento de la población y los recursos naturales agotables. Sin embargo, dicho enfoque difícilmente se le analiza e incluso se le tiende a calificar como parte de una filosofía ecologista pesimista (Samuelson y Nordhaus, 1996) o como una política de antinatalidad. 
En otras palabras, el tema de los desastres de origen antrópico resulta difícil de enfocar, pues se excluye del discurso este aspecto demográfico y la responsabilidad del ser humano en esta situación de amenaza, cayéndose fácilmente en intervenciones aisladas e ineficaces. Siguiendo con el ejemplo, en este contexto se podría defender el derecho de los niños y las niñas a la salud; pero por otro lado, no se analizan las razones por las cuales se da un consumo de agua contaminada, que les podría causar problemas gástricos, parasitosis o enfermedades contagiosas que los llevarían incluso a la muerte, entre otros problemas. Estas contradicciones merecen profundizarse en una educación ambiental que integre las causas del problema desde diferentes áreas del conocimiento, para no seguir haciendo esfuerzos aislados que no atacan el origen de las situaciones catastróficas.

Coca (2005:1) plantea el asunto cuando afirma que "el tema de los riesgos y los desastres está claramente ligado con problemas de desarrollo, es decir, con desequilibrios entre el ambiente social, el ambiente construido y el ambiente natural de un territorio que aumentan la susceptibilidad y la exposición a diferentes tipos de amenazas". Además, la autora agrega que el crecimiento acelerado de la población es uno de los factores que intervienen para aumentar las amenazas y "la vulnerabilidad socialmente construida".

Por otra parte, desde el punto de vista económico, parece que se ha llegado a un sistema de producción que ha perdido su razón de ser. En otras palabras, la búsqueda del bienestar de la población ha llevado contradictoriamente en un nivel de insostenibilidad que amenaza tan anhelada condición. Por consiguiente, desde una perspectiva de la educación ambiental, surge la necesidad de revisar los patrones de consumo y de explorar sistemas alternativos de producción limpia, aspectos insoslayables en los planes formativos de una educación ambiental más integral.

Cabe profundizar en este artículo el ejemplo concreto del manejo del recurso hídrico. Algunos se preguntarán: ¿qué relación tiene el manejo del agua con los desastres de origen antrópico, además de las inundaciones o de las sequías?. Para evitar estas situaciones, se requiere respetar las áreas de recarga, por lo que la protección de las mismas enfrentaría las acciones de quienes defienden intereses económicos muy fuertes, pues tratan de acaparar, precisamente, esas zonas, para garantizarse una venta futura del líquido. El agua, al ser un elemento insustituible para mantener la vida, promete negocios de la más alta rentabilidad desde la perspectiva economicista antes mencionada. Por otro lado, se presentan amenazas del recurso con una tala insostenible de árboles para explotar la madera o edificar zonas sin ninguna 
planeación urbana. Se suma el aumento del uso de esas tierras para cultivos en los que se utilizan insecticidas altamente tóxicos y que contaminan el agua, pero que garantizan una alta producción de alimentos.

Desde el punto de vista de una educación ambiental integral, no se podría dejar de analizar este aspecto económico, que se constituye en una amenaza y que hace insuficientes y descontextualizados aquellos esfuerzos educativos que buscan enseñar a los niños y a las niñas a no desperdiciar el agua o el saber conducirse ante inundaciones en las localidades de alto riesgo.

Así, el que gran cantidad de comunidades en los diferentes países no dispongan de agua potable, entre estos algunas poblaciones de Costa Rica, ni que se tenga la certeza de que se contará con el abastecimiento necesario de agua en los próximos años, implica analizar intereses políticos, económicos y religiosos muy fuertes; existirán quienes harán todo lo posible para obstaculizar un debate al respecto. Sin embargo, pareciera que no hay alternativa y la educación ambiental tendrá que tomar esos rumbos si se quiere ser eficaz en la prevención de desastres.

Sin embargo, es importante mencionar aquí aquellos intentos teóricos que se están presentando para ir incluyendo, como eje transversal una perspectiva ecológica, cuando se menciona el desarrollo humano. Por ejemplo, en algunas definiciones de inteligencia se incorpora la relación que la persona establece con su medio ambiente. No cabe duda entonces de la necesidad de estimular el desarrollo de los niños y las niñas en lo que Howard Gardner ha llamado la inteligencia naturalista (Farben, s.f.). Esto demanda crear nuevas estrategias de estimulación, dejándose de considerar el desarrollo humano de los niños y las niñas como simplemente la aparición de etapas secuenciales que se presentan en forma independiente del desarrollo social, económico o cultural.

Interesa destacar, con el ejemplo anterior, que desde el punto de vista de la educación ambiental vigente, se aprecia un fuerte desconocimiento de las causas del problema o una falta de preocupación o compromiso ante el hecho de que una mayoría de la población será afectada. Esto se podría enfocar desde la perspectiva de un comportamiento moral o ético, pero que no es el objetivo de este artículo.

Desde una perspectiva economicista, hemos aprendido que se garantiza el bienestar futuro de los hijos, si se les proporciona una educación que les permita el mejor ingreso posible, o con una propiedad donde puedan vivir en el futuro, pero se excluye del pensamiento el hecho de que los hijos y los nietos, que ya nacieron, tendrán dificultades para disponer de agua, si se sigue 
con los patrones de conducta actuales. Dentro de este esquema, no importa si hay desastres tales como las inundaciones que afectan a quienes viven en lugares vulnerables. En otras palabras, es un "no me importan los demás", que lleva a pensar en cómo se promueve, desde el punto de vista educativo, ese aprendizaje de tal indiferencia y conformidad, tema clave para un enfoque de una educación ambiental más integral (Flen-Febers, 2001).

La educación ambiental pasa a ser una tarea inconclusa, a pesar de que en el sistema educativo se menciona como una prioridad, desde hace varios años. Quizás este avance tan lento se deba a que no se ha profundizado lo suficiente en cómo cambiar esos tipos de pensamiento que se refieren a una visión del comportamiento humano y del medio ambiente, muy particulares. Se mantiene así una visión centrada en una prepotencia del ser humano ante otras formas de vida, un aprendizaje de conocimientos aislados, que pierden de perspectiva que el bienestar se deriva de esa armonía con la naturaleza y no de una explotación insostenible que provoca los desequilibrios de carácter irreversible. Se conceptúa la Naturaleza como algo que debe dominar, explotar para su beneficio, sin ver la interdependencia de las partes que forman un todo del que se es parte.

En una oportunidad escuché el comentario: "no es que hay mucha gente en el mundo, el problema es de distribución de los recursos". Esta frase hace pensar que en el fondo existe un pensamiento que parte de la premisa de que los recursos naturales son inagotables, como se enseñaba hace tan sólo treinta años. Bajo esta perspectiva, no se reconoce que los recursos son agotables, en especial si se continúa con el incremento actual de la población y se niega así el impacto de la huella ecológica que deja cada persona, al consumir los diferentes productos durante todo un día y a lo largo de su vida.

Como reflexión final, se podría anotar que la construcción de una educación ambiental más integral e interdisciplinaria permitirá evitar algunos desastres de origen antrópico, en la medida que integre y posibilite un análisis de esas formas de pensamiento, principalmente de corte económico y religioso, que mantienen la separación de aspectos tales como el nivel de consumo de los recursos naturales y el crecimiento demográfico.

Así, está pendiente la tarea de revisar esas formas de pensamiento que no enfocan las causas y se quedan a nivel de las consecuencias, por lo cual las intervenciones dejan de ser preventivas y no permiten reconocer que somos los únicos responsables de provocar los desastres de origen antrópico. En otras palabras, que los mismos no se presentan porque Dios asílo quiere, sino que son producto de una negligencia derivada de esos pensamientos que nos impide actuar en forma diferente. 
Cabe mencionar que esas formas de explicar la realidad se plasman en los libros de texto y en aquellos recursos didácticos que se utilizan en las escuelas, colegios y universidades. Por consiguiente, se podría empezar con un análisis del uso que se hace de los animales y de las plantas que decoran los centros educativos, las cuales en algunos casos se dejan morir, pues los niños y las niñas no aprenden a cuidarlas, especialmente si el docente tampoco se ha formado en valores que le permiten una concepción diferente de la vida. Se aprende, entonces, un menosprecio por lo que no es humano, lo que lleva a que la flora y la fauna sólo sean consideradas por su utilidad para los seres humanos. Dentro de esta perspectiva, se tiende a pensar que los animales y las plantas están allí para explotarlos y además se dice que no piensan, que no sienten, lo que les convierte en seres inferiores. La autora Armellle Le Bras-Chopard (2003) realiza un análisis profundo de cómo hemos clasificado ciertos animales en la dinámica del poder, lo cual merece una atención desde el punto de vista epistemológico. Revisar este tipo de pensamiento, parece ser un asunto clave en una educación ambiental integral.

Se une un abordaje muy particular del asunto en los medios de información, en los cuales se refuerza una idea de que la Naturaleza es aquel monstruo que hay que dominar, pues se dice que esta se ensaña contra los individuos, soslayándose así la necesidad de explicar la responsabilidad del ser humano en ese acontecer. A lo anterior se asocia un bombardeo de películas que van insensibilizando el problema y nos hacen creer que el desastre final es algo irremediable, pues nos muestran, por ejemplo, ciudades como Nueva York en el año 2065 en el que no existirá rastro de vida. No se puede pensar en una educación ambiental que no analice críticamente tales mensajes y formas de pensamiento.

Es importante mencionar aquí la necesidad de una revisión de la educación para la sexualidad integral, en la cual se asocie la protección ambiental, los desastres de origen antrópico y un crecimiento no planificado de la población. En la actualidad, todavía parece una herejía el pensar que el crecimiento geométrico de la población conlleva una disminución de los recursos y un aumento de la contaminación. Si bien se pueden buscar formas de producción limpia y variar los patrones de consumo, el exceso de población demanda bienes y servicios de forma insostenible.

En este contexto donde surgen tantas interrogantes, resulta descabellado defender los abusos a la Naturaleza; pareciera que lo que hay de fondo es que no importan los semejantes, quienes mueren por condiciones como las antes descritas, en una sociedad que olvidó que amar al prójimo es una de 
las reglas básicas para la convivencia. Así, dentro de un pensamiento individualista que hace que no nos importe aquel que sufre, menos nos importarán los otros seres vivos que nos rodean, y menos entenderemos que somos una parte más de todo un sistema.

En resumen, se requiere analizar ciertos pensamientos religiosos y económicos que parecen ir de la mano al fomentar o mantener una cultura de indiferencia e impotencia. En ese contexto, no cabe duda de que una educación ambiental más interdisciplinaria puede romper epistemológicamente con tales discursos, lo que permitirá reconocer nuestra participación en el problema y dejar de pensar que Dios o la Naturaleza nos mandan los desastres de origen antrópico. 


\section{REFERENCIAS}

Alfaro, Tomás. Los huracanes. Monstruos de agua y viento. Zurquí. Suplemento Educativo de La Nación. 23-11-2005, pp. 12-13.

Comisión Nacional de Emergencias. Primer Encuentro Nacional sobre Educación y Capacitación en Gestión de Riesgo. 15 y 16 de noviembre.

Coca, Patricia (2005) Dimensión pedagógica y curricular de la gestión del riesgo.

San José, Costa Rica: Primer Encuentro Nacional sobre Educación y Capacitación en Gestión de Riesgo. Comisión Nacional de Emergencias.

Farben Grupo (s.f.) Inteligencias Múltiples. Nota técnica. Editorial Norma.

Field, Barry C. (1998) Economía ambiental. Una introducción. Colombia: McGrawHill Interamericana, S.A.

Flen-bers, Melba (2001) Actitud del docente preescolar frente a la educación ambiental. Instituto Universitario de Educación Especializada, Educación, Mención Preescolar. Trabajo especial de Grado. Maracaibo Estado Zulia.

www.monografias.com/trabajos14/docentepresc.shtml

Foro Internacional de ONG's y Movimientos Sociales (1993) Construyendo el futuro. Tratados alternativos de Río'92. San José, Costa Rica: Porvenir: CECADE: Consejo de la Tierra: Universidad Nacional.

Guerrero, Francisco (s.f.) Leyendo a los clásicos. Howard Gardner. Revista Digital de Educación y Nuevas Tecnologías. www.monografias.com

Gutiérrez, Francisco y Prado R., Cruz (s.f.) Ecopedagogía y Ciudadanía Planetaria. Heredia, Costa Rica: Editorialpec 97.

III Conferencia Salud Ocupacional y Ambiental en las Américas (2005) Alajuela, Costa Rica.

Jengich Buck, Alexa (1999) El pensamiento del movimiento ecologista en Costa Rica. Ensayo sin publicar, producido en el curso Tendencias del pensamiento político y social en América Latina II. Programa de Doctorado en Estudios Latinoamericanos con mención en Pensamiento Latinoamericano, Universidad Nacional.

Le Bras-Chopard, Armellle (2003) El zoo de los filósofos. Madrid, España: Santillana Ediciones Generales, S. L.

Merleau-Ponty, C. y Girardet, S. (s.f.) Tu pueblo es tu planeta. España: UNESCO.

Mires, Fernando El discurso de la naturaleza. Ecología y política en América Latina. San José, Costa Rica: Editorial DEI.

Mora, Arnoldo. Ecología, ética y filosofía. Praxis. Revista del Departamento de Filosofía, Universidad Nacional. 43-44, 1975: 19-26

Mora, Eduardo (1998) Naturaleza, quéherida mía. Ambientalismo, Estado, natura y mercado. Heredia, Costa Rica: Universidad Nacional: Ambientico Ediciones. 
Murillo Rodríguez, Carlos (2000) Comercio y ambiente. Ensayos críticos. Heredia, Costa Rica: EUNA.

Neuburger, Alicia B. Prevención e intervención psicosocial en situaciones de desastre. Praxis. Revista del Departamento de Filosofía, Universidad Nacional. 43-44, 1975: 247-252

Proyecto SIMED (1996) Reflexiones y prácticas sobre la educación ambiental, el enfoque de género y la sexualidad en las escuelas líderes. San José, Costa Rica: Ministerio de Educación Pública. UNESCO - Países Bajos.

Romero Sepúlveda, Rodia. El desarrollo sostenible: un concepto polémico. Praxis. Revista del Departamento de Filosofía, Universidad Nacional. 43-44, 1975: 155-166

Salas B., Sonia (s.f.) Desarrollo Regional Sustentable. Definición y percepción académica. Chile: Universidad de La Serena, Proyecto Capacitación en Desarrollo Regional Sustentable.

Samuelson Paul A. y Nordhaus William D. (1996) Economía. España: McGraw Hill Sternberg, Robert J. y Lubart, Todd I. (1997) La creatividad en una cultura conformista. Un desafio a las masas. Barcelona, España: Editorial Paidós.

Waksman, Vera y Kohan Walter (2000) Filosofía con niños. Aportes para el trabajo en clase. Argentina: Ediciones Novedades Educativas. 